\title{
Communication
}

\section{Low-cost, pseudo-Halbach dipole magnets for NMR}

\author{
Michael C.D. Tayler ${ }^{\mathrm{a}, \mathrm{b}, *}$, Dimitrios Sakellariou $^{\mathrm{c}}$ \\ a Magnetic Resonance Research Center, Department of Chemical Engineering and Biotechnology, University of Cambridge, Cambridge, UK \\ ${ }^{\mathrm{b}}$ Department of Physics, University of California, Berkeley, CA 94720, USA \\ ${ }^{\mathrm{c}}$ NIMBE, CEA-CNRS, Université Paris-Saclay, CEA Saclay, 91191 Gif-sur-Yvette Cedex, France
}

\section{A R T I C L E I N F O}

Article history:

Received 7 February 2017

Revised 1 March 2017

Accepted 2 March 2017

Available online 6 March 2017

\section{Keywords:}

Nuclear magnetic resonance (NMR)

Permanent magnets

Low-field NMR

Zero-field NMR

\begin{abstract}
A B S T R A C T
We present designs for compact, inexpensive and strong dipole permanent magnets aimed primarily at magnetic resonance applications where prepolarization and detection occur at different locations. Lowhomogeneity magnets with a $7.5 \mathrm{~mm}$ bore size and field up to nearly $2 \mathrm{~T}$ are constructed using low-cost starting materials, standard workshop tools and only few hours of labor - an achievable project for a student or postdoc with spare time. As an application example we show how our magnet was used to polarize the nuclear spins in approximately $1 \mathrm{~mL}$ of pure $\left[{ }^{13} \mathrm{C}\right]$-methanol prior to detection of its highresolution NMR spectrum at zero field (measurement field below $10^{-10} \mathrm{~T}$ ), where signals appear at multiples of the carbon-hydrogen spin-spin coupling frequency ${ }^{1} J_{\mathrm{CH}}=140.7(1) \mathrm{Hz}$.
\end{abstract}

(c) 2017 The Author(s). Published by Elsevier Inc. This is an open access article under the CC BY-NC-ND license (http://creativecommons.org/licenses/by-nc-nd/4.0/).

\section{Introduction}

Nuclear magnetic resonance (NMR) spectra are often desired at the highest available strength of magnetic field, $B$, since detection sensitivity is proportional to $B^{7 / 4}$ and the resolution of chemical shifts (subject to field homogeneity) scales linearly with $B$. The highest static fields available today are up to $23 \mathrm{~T}$ using persistent superconducting magnets, which when coupled with optimized signal detection achieve sensitivities in the low picomole $\left(10^{-12}\right.$ moles) range.

The design and construction of a single NMR magnet to meet the demands of high field strength, part-per-billion homogeneity, high stability and minimal stray field, however, is an expensive undertaking and at the strongest fields the final product is neither compact or mobile [1,2]. For a significant reduction in the power required for NMR and an improved mobility of the instrumentation, the use of rare-earth permanent magnet assemblies (up to $1.5 \mathrm{~T}$ ) has become popular. Sintered neodymium-iron-boron $(\mathrm{NdFeB})$ magnets have revolutionized a number of technologies, including electric motors and recently the design of NMR magnets, due to their high values of remanence and coercivity that allow for very strong and compact magnet structures with very good resistance to demagnetization [3]. Very soon after NdFeB was introduced in the early 1980s, Klaus Halbach was one of the first to

\footnotetext{
* Corresponding author at: Magnetic Resonance Research Center, Department of Chemical Engineering and Biotechnology, University of Cambridge, Cambridge, CB3 OAS, UK.

E-mail address: mcdt2@cam.ac.uk (M.C.D. Tayler).
}

capitalize on these properties, proposing an ingenious way to design and build magnets producing multipolar fields [4]. The cylindrical structure and later the spherical structure [5] allowed dipolar magnetic fields exceeding $2 \mathrm{~T}$ to be produced. Since then, several research groups and companies have refined Halbach's structures and together with shim coils magnets up to around $1.5 \mathrm{~T}$ can be obtained with homogeneity to better than $0.05 \mathrm{ppm}$, allowing for the resolution of chemical shifts and nuclear spinspin couplings in the measured NMR spectra [6-14]. The sensitivity at $1.5 \mathrm{~T}$ is around $10^{-7}{ }^{1} \mathrm{H}$ moles, sufficing for most analyses of solution-state samples in the synthetic chemistry laboratory and at a price point that can enter the teaching laboratory.

In the present work we discuss the design, assembly and application of permanent magnet arrays where field strength and ease of construction are prioritized over magnetic field homogeneity. These are aimed at magnetic resonance applications that do not require sub-ppm field uniformity, for example nuclear prepolarization of samples prior to remote detection of the signal in earth's field [15] or ultra-low field $[16,17,27]$. In some cases the magnetic field homogeneity may be acceptable for in situ highresolution NMR spectroscopy of small-volume samples, which are less susceptible to field inhomogeneity $[18,19]$, or in conjunction with RF pulse sequences that correct for the inhomogeneity [20].

\section{Design of pseudo-Halbach arrays}

The original theoretical design of Halbach for multipolar permanent magnets assumed an infinitely long cylindrical structure 
having a continuous distribution of orientation-dependent, fixedmagnitude magnetizations. Practical implementations, however, must have finite length and allow for a segmented assembly. Most high performance assemblies use wedge-shaped magnet blocks, which require special magnet grinding and are best produced and assembled by experts. A few pseudo-Halbach dipole magnets for NMR have been built using clusters of identical permanent magnets that can be purchased, such as cylinders [6], cubes [12] and other regular prisms [21-23]. This last approach gave rise to the "Mandhalas" family of magnets. However, in most cases these assemblies are designed for optimal magnetic field homogeneity at the expense of magnetization density, and sometimes to constrain the magnetization density to give a "convenient" value of nuclear Larmor frequency or for some other reason, e.g. to facilitate sample access $[14,24]$. Our effort here was to design an ultra-compact dipole magnet inspired by the original Halbach design, which can be produced using many identical permanent magnet pieces, similar to the Mandhalas concept, and has an even higher magnetization density.

The initial design was based on the availability of parallelepipedic $\mathrm{NdFeB}$ magnet blocks with dimensions of $2.00 \times 0.500 \times 0.500$ in. $^{3}$ each magnetized through the 0.5 in. thickness (CMS Magnetics: N52 grade, remanent field $B_{r}=1.44 \mathrm{~T}$, $\mathrm{Ni}-\mathrm{Cu}-\mathrm{Ni}$ plating, dimensions accurate to +0.002 in., $\$ 4.50$ per block, $\$=$ USD, 1 in. $=25.4 \mathrm{~mm}$ exactly). In anticipation of holding the NMR sample inside the magnet array within a standard $5 \mathrm{~mm}$ outer-diameter glass NMR tube, a rectangular cross section of $0.5 \mathrm{in}$. by $0.3 \mathrm{in}$. $(7.5 \mathrm{~mm}$ ) was reserved for non-magnetic material. Exact numerical calculations were then performed using analytical formulae from the literature [11] to determine the maximum field strength obtainable in assemblies of two, four, eight and twenty-four of the block units surrounding the void (or "bore"), assuming perfectly rigid and uniform magnetizations over the block volumes.

Demagnetization within permanent magnet arrays can become an important factor when high magnetic fields are involved. Magnetic materials are also characterized by a coercivity field $\left(H_{c}\right)$ value [25], which indicates the resilience of the material to demagnetization in the presence of an external magnetic field at a given temperature. Temperature and mechanical stress are important factors as these govern the dynamics of magnetic domain shape and orientation [26]. Even slight increases in temperature may lead to a higher plasticity and values of coercivity subject to demagnetization. One has to be careful when designing a very strong permanent magnet to compute the values of the magnetic field everywhere in space and in particular inside the magnetic material - during assembly as well as for the final structure [29] - in order to avoid moving the working point of the material beyond the demagnetization "shoulder". Going past this point leads to an irreversible loss of magnetization, which in turn will reduce the produced magnetic field and induce substantial field inhomogeneities. For this reason, the magnet should also not be exposed to temperatures outside its intended operating range.

To obtain a practical design where field is maximized but the effects of demagnetization are reduced, we searched for the optimal arrangement for two grades of magnet material when using more than 8 magnet blocks. For the second material, N48SH grade was chosen $\left(B_{r}=1.37 \mathrm{~T}, \$ 10\right.$ per block), since the remanence value is only around $5 \%$ less than N52 but the coercivity is approximately 2 times greater. The magnet orientations producing the strongest total field within the array structure are illustrated in Fig. 1. The field values at the geometric centers of each array are summarized in Table 1. The calculated value of field in all four magnet arrays given on the first line ignores demagnetization effects and is calculated using a rigid-magnetization model: magnetization is uniform over the material volume, and constant through it, equal to the

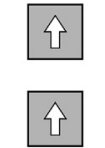

2 blocks

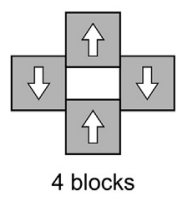

Material:

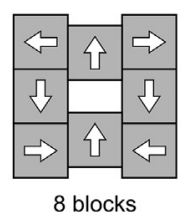

8 blocks

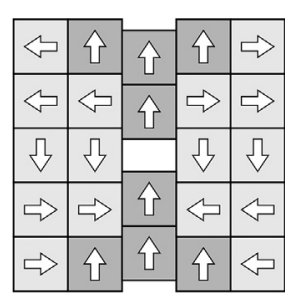

24 blocks
Fig. 1. Cross sections of the Pseudo-Halbach permanent magnet assemblies involving 2, 4, 8 and $24 \mathrm{NdFeB}$ magnet blocks. Arrows represent the axis of magnetization in the material. The first three structures use a single type of material, while the last one uses two types to reduce demagnetization effects.

Table 1

Calculated and measured central magnetic fields (in tesla) for the arrays shown in Fig. 1 calculated using $\mathrm{N} 52$ and $\mathrm{N} 48 \mathrm{SH}$ materials data at room temperature. The central gap measures $0.5 \mathrm{in}$. $(12.7 \mathrm{~mm})$ by $0.3 \mathrm{in}$. $(7.6 \mathrm{~mm})$

\begin{tabular}{lcccc}
\hline No. of blocks & 2 & 4 & 8 & 24 \\
\hline Magnetization & & & & \\
Rigid & 0.651 & 1.031 & 1.482 & 2.000 \\
Non-rigid & 0.629 & 1.007 & 1.445 & 1.950 \\
Experimental & - & - & 1.38 & 1.93 \\
\hline
\end{tabular}

value of the remanence $B_{r}$. This situation is not realistic since in practice the material is in the presence of a non-zero magnetic field created by the other magnetic materials, as well as by itself. The magnetization thus is less than the remanence and follows the demagnetization curve. In this realistic case we speak about a "non-rigid" magnetization, which the value of magnetic field on second line takes into account. For the 8- and 24-block arrays, constructed as detailed below, the experimentally measured values of the magnetic field are also given.

\section{Construction}

Casings to contain the 8- and 24-block magnet arrays were fabricated from 6061 aluminum in the student machine shop of the UC Berkeley physics department. A bandsaw, a lathe, a milling machine and a simple hand-finishing tools were used to build a structure with outer dimensions $3.00 \times 3.00 \times 3.00$ in. $^{3}$ for the 8 block magnet. A $4.00 \times 4.00 \times 3.00$ in. $^{3}$ version was built for the 24-block magnet. The final structures including the NdFeB blocks weighed a total of $1.3 \mathrm{~kg}$ (8-block version) and $3.0 \mathrm{~kg}$ (24-block version) respectively. Scale drawings for the constructed parts have been provided free-of-charge in the online Supplementary Information.

The Supplementary Information contains detailed instructions for assembling the magnet arrays. All of the array structures shown in Fig. 1 could be assembled by hand, without the need for machine presses, glue or clamping tools and without compromising safety. Nevertheless, the complexity and difficulty of the task increased rather quickly with the size of the array.

Fig. 2 summarizes the stages involved in the assembly of the 24block magnet array. The first stage is to install the two central magnets that contribute most towards the final pseudo-dipolar field, i.e. whose magnetization is oriented along the axis of the dipole (Fig. 2(a)). The central part is a 0.5 in. outer diameter, 0.295 in. inner-diameter, 3-in. long aluminum tube with the central 2-in. length of the exterior machined down give two flat, parallel slots separated by a thickness of $0.3 \mathrm{in}$. These slots accommodate the magnet blocks to give the desired central void. In the next stage, the magnet blocks inserted are those which partially attract to 

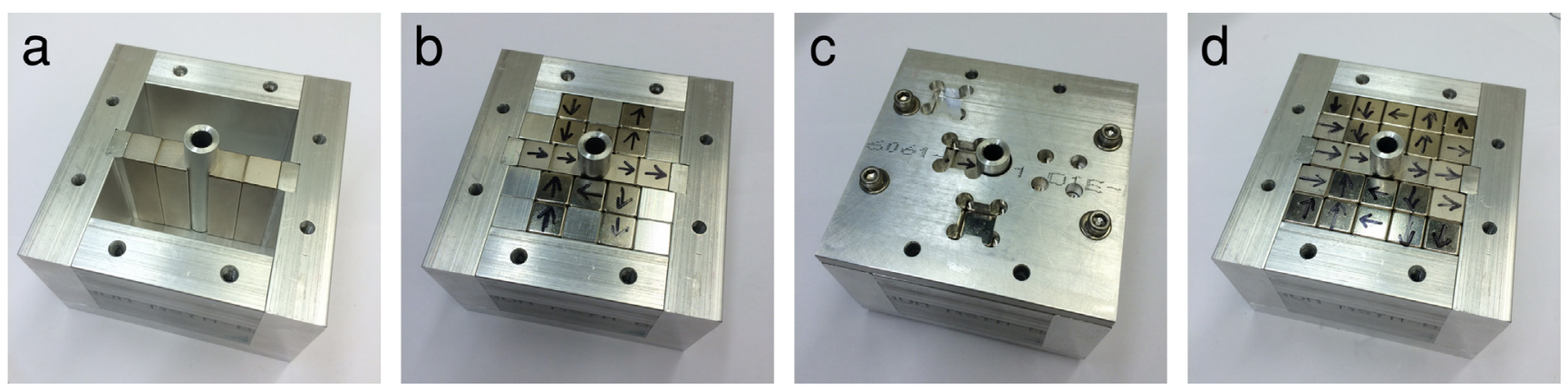

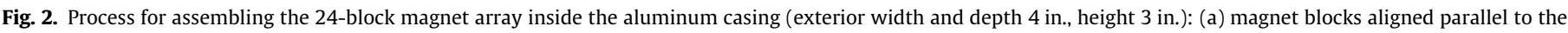

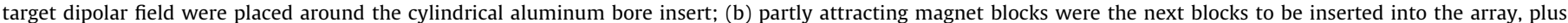

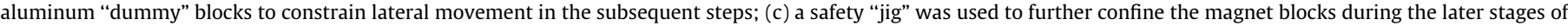

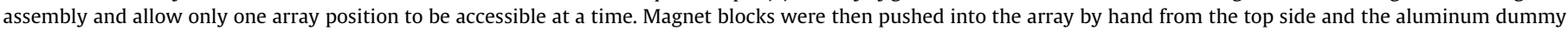

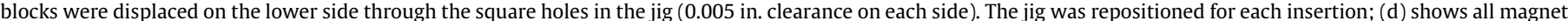
blocks in their final positions before the top and bottom covers were fitted and screwed in place.

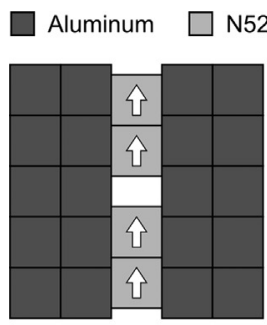

$0.85 \mathrm{~T}$

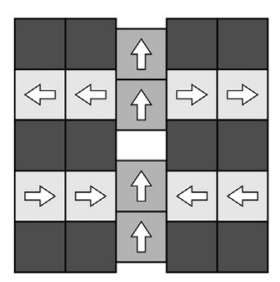

$1.30 \mathrm{~T}$

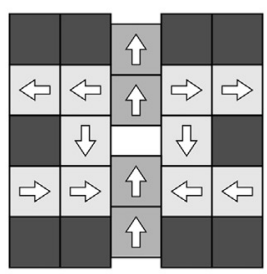

$1.65 \mathrm{~T}$

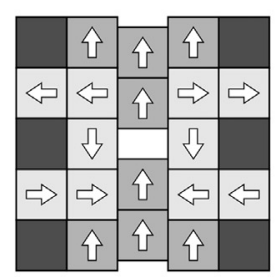

$1.75 \mathrm{~T}$

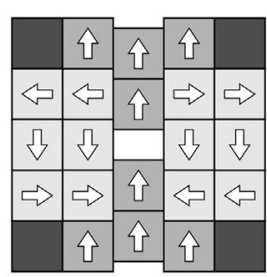

$1.85 \mathrm{~T}$

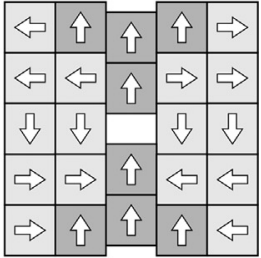

$1.95 \mathrm{~T}$

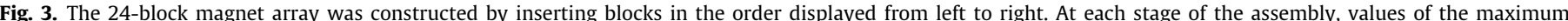

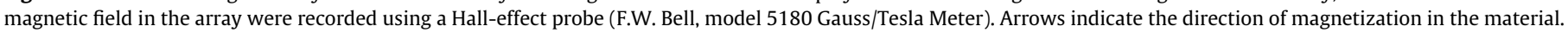

those already in place and are easily inserted by hand. Fig. 2(b) shows how these blocks are kept in their correct position by filling also the remainder of the array with non-magnetic "dummy" blocks made from aluminum. The dummy blocks are approximately $0.002 \mathrm{in}$. oversize in width and depth to assist the next stage of assembly. The following task is also assisted by holding the magnet casing in a vise. Working outwards from the center of the array, remaining blocks are pushed into place by hand, one at a time. As one block is pushed into the array from one side, the aluminum dummy block is displaced out of the other side. This method ensures there is zero free space for other magnet blocks to move around. It is necessary use a jig like the one shown in Fig. 2(c) to avoid potentially very unsafe (and unpredictable) high-speed ejection of other magnet blocks from the array due to release of the intense repulsion force that is contained. In the Supplementary Information, we provide one design of jig that will assist a safe assembly of the whole 24-block array. When all of the blocks have been placed into position (Fig. 2(d)), the jigs are removed and 0.5 in. thick covers are secured to the top and bottom of the side walls using national-fine 10-32 hex-head screws. The whole assembly procedure takes about $30 \mathrm{~min}$. It is recommended that a safety notice (e.g. a sticker, an engraving or an etch) is placed on the casing to inform users of the hazards associated with strong magnetic fields.

\section{Performance of the magnet arrays}

The strength of the magnetic field was measured using a Halleffect probe (F.W. Bell, model 5180). The peak magnetic field was measured during each stage of the assembly for the 24-block magnet and the values are shown in Fig. 3. In the final structures the field was also measured at 1/8-in. intervals along the magnet bore

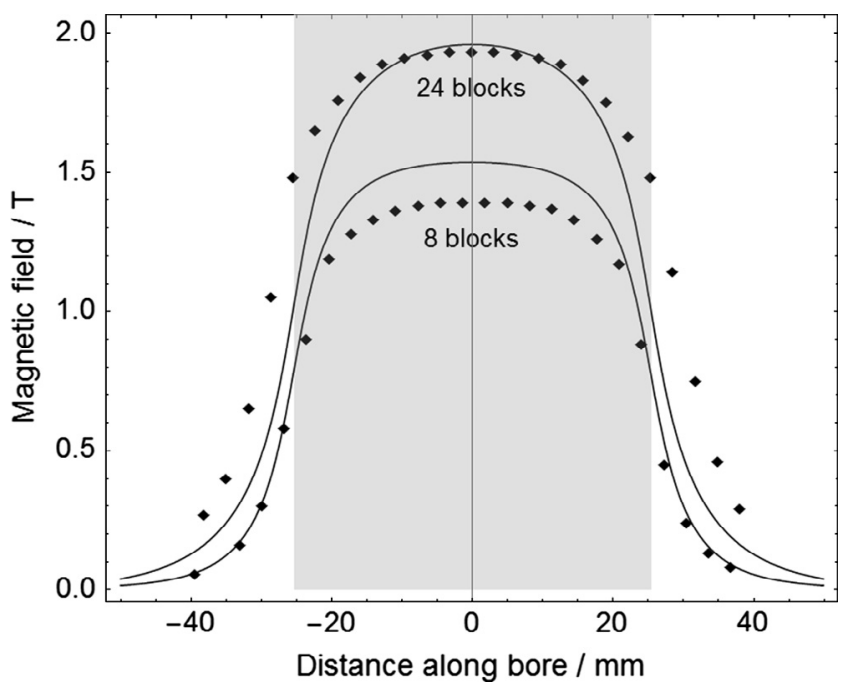

Fig. 4. Field strengths of the 8-block and 24-block magnet arrays versus distance along the bore axis. The diamonds correspond to experimental measurements using a Hall-effect probe, while the solid lines correspond to the result of advanced numerical modeling of the magnet array, given the materials data provided (see Supplementary Information).

and the values are plotted in Fig. 4. The calculated profiles for the magnet arrays are plotted on the same figure as solid lines. The experimental data reproduce only roughly the theoretical predictions, even when advanced modeling including demagnetization was used. Since the magnetic pieces were not sorted beforehand in order to have controlled characteristics [11], significant variations in magnitude and direction of magnetization could be 
present in some blocks: in our experience, $\pm 10^{\circ}$ in orientation, and $\pm 5 \%$ deviations in field magnitude can be expected for magnets blocks that are bought and used without selection. We expect these effects to decrease for magnetic structures that require a large number of pieces, because on average such deviations are damped out, and this is why we believe that the overall profile from the 24-block magnet corresponds better to the theoretical model, compared to the 8-block one. For the 8-block magnet, the field at the center measured at room temperature was $1.38 \mathrm{~T}$; this is lower than the predicted $1.445 \mathrm{~T}$, perhaps due to demagnetization in the N52 material. For the 24-block version the maximum field was $1.93 \mathrm{~T}$ (1.96 $\mathrm{T}$ predicted). The magnetic field was greater than $95 \%$ of the peak values at 0.5 in. either side of the central maximum, which equals a volume of 0.068 cubic inches $\left(1.1 \mathrm{~cm}^{3}\right)$.

Dipole Halbach magnets provide an additional interesting feature, namely a very small stray magnetic field. Only for the ideal design of the infinite Halbach cylinder is the magnetic field outside strictly null, but even in practice it is usually extremely small because of the very efficient field line confinement inside the magnet structure. In our magnet design, however, since we have used only two possible orientations, the question about the quality of the field confinement remains open. We thus have calculated the theoretical values of the stray magnetic fields for the 8-block and 24-block assemblies and we represent its magnitude in Fig. 5 as 3D surfaces of $|B(x, y, z)|$, orienting the $z$ axis of the plot with the symmetry axis of the magnet bore and orienting the $y$ axis with the net magnetic dipole. The 5 gauss $(|B|=0.5 \mathrm{mT})$ surface is well within a spherical volume having a radius of approximately $12 \mathrm{~cm}$ and $25 \mathrm{~cm}$ for the 8- and 24-block assembly, respectively. Experimental measurements performed outside of the aluminum frame agree with these theoretical predictions. At the limits of the casing the peak stray fields $|B|$ are much stronger: (8-block assembly) $x y$ faces, $0.1 \mathrm{~T}$; $y z$ faces, $0.012 \mathrm{~T}$; $x z$ faces, $0.025 \mathrm{~T}$; (24-block assembly) $x y$ faces, $0.3 \mathrm{~T}$; $y z$ faces, $0.04 \mathrm{~T} ; x z$ faces, $0.08 \mathrm{~T}$. This feature makes our assemblies magnetically compatible with other instrumentation reducing risks for hazardous operations. Nevertheless, stray field remain a hazard; as a reference, the damage threshold for a high-quality magnetic card (e.g. an ATM or credit card) is around $0.03 \mathrm{~T}$.

We tested the magnet within an "ex-situ" NMR experiment by using it to polarize the nuclear spins in a sample of $0.1 \mathrm{~mL}\left[{ }^{13} \mathrm{C}\right]-$ methanol (Sigma Aldrich Isotec) contained inside a $50 \mathrm{~mm}$ long, $5 \mathrm{~mm}$ o.d. flame-sealed NMR tube. The sample was initially held in the field of the magnet, then shuttled into a spin-exchange relaxation-free (SERF) rubidium magnetometer of sensitivity $10-$ $20 \mathrm{fT} / \mathrm{Hz}^{-1 / 2}$ to detect evolution of the polarization under the chemically specific ${ }^{1} \mathrm{H}^{-13} \mathrm{C} \mathrm{J}$ coupling at zero field $\left(|B|<10^{-10} \mathrm{~T}\right)$ $[27,28]$. The sample and the magnet were positioned above the Mu-metal shield of the magnetometer (Twinleaf LLC) as illustrated in Fig. 6 with a $10 \mathrm{~cm}$ distance between the shield and the magnet's aluminum casing. A $7.5 \mathrm{~mm}$ o.d. and $6.5 \mathrm{~mm}$ i.d. epoxy tube (TAP plastics) was used to guide the vertical transport of the sample tube between the high-field and low-field regions. The sample was shuttled down to the magnetometer using gravity and was raised, for re-polarization, by activating a solenoid valve that opens the top of the tube to a suction (low-vacuum) line. When the sample comes to rest inside the magnetic shield, a DC pulse was applied to excite spin coherence [28]. The magnetometer signal, which is proportional to the total sample magnetization, was digitally sampled for a period of $10 \mathrm{~s}$ and Fourier transformed into the high-resolution zero-field spectrum shown on the Figure inset. The peaks in the spectrum correspond to the frequencies of the one-bond ${ }^{13} \mathrm{C}-{ }^{1} \mathrm{H}$ spin-spin coupling, extracted as $J_{\mathrm{CH}}=140.7(1)$ $\mathrm{Hz}$ and $2 \times J_{\mathrm{CH}}=281.4(1) \mathrm{Hz}$. This experiment was repeated after replacing our magnet with a commercial Halbach array $(2.05 \mathrm{~T}$,
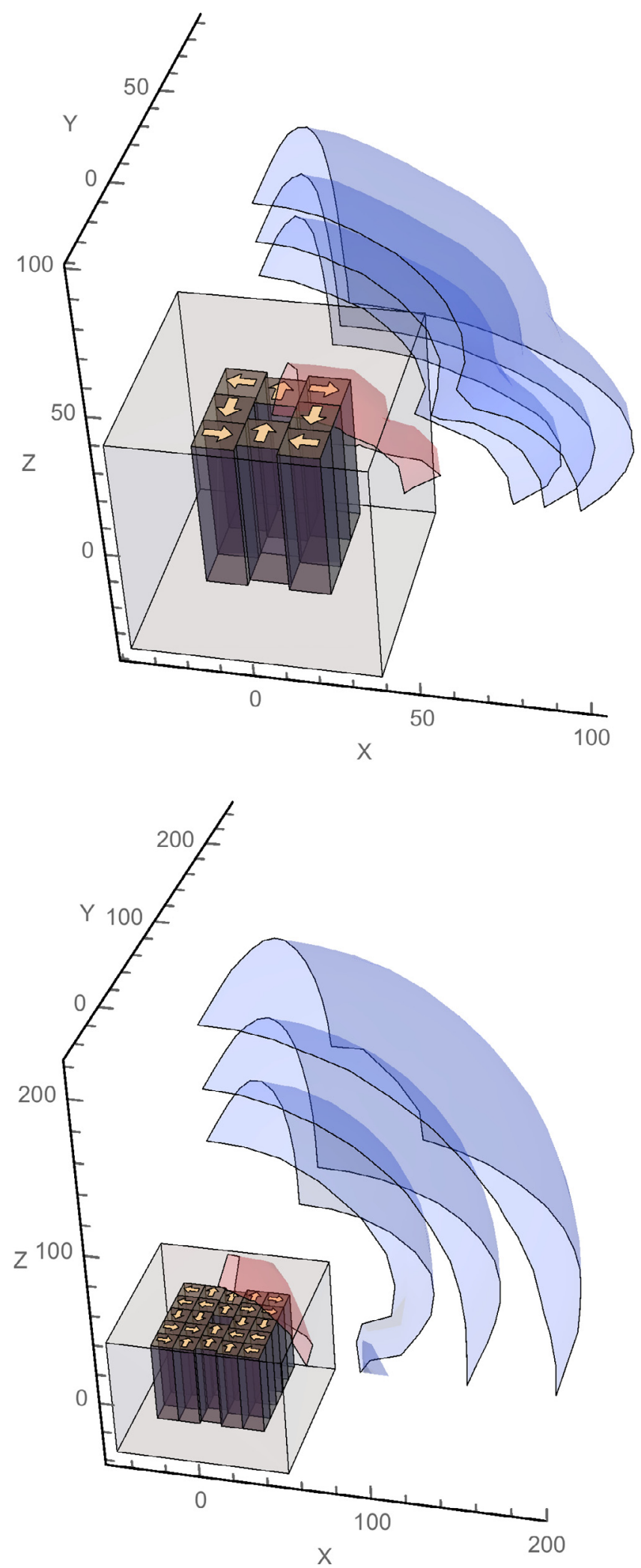

Fig. 5. Calculated stray fields for the 8-block and 24-block magnet arrays (upper and lower plots, respectively). The contour surfaces represent field strengths of 5 , 10, 20 gauss (in blue) and 1000 gauss (in red). (For interpretation of the references to color in this figure legend, the reader is referred to the web version of this article.)

BFlux Technologies model RHR-2T-10), which was composed of 8 wedge-shaped magnet segments around a $10 \mathrm{~mm}$ diameter bore. 

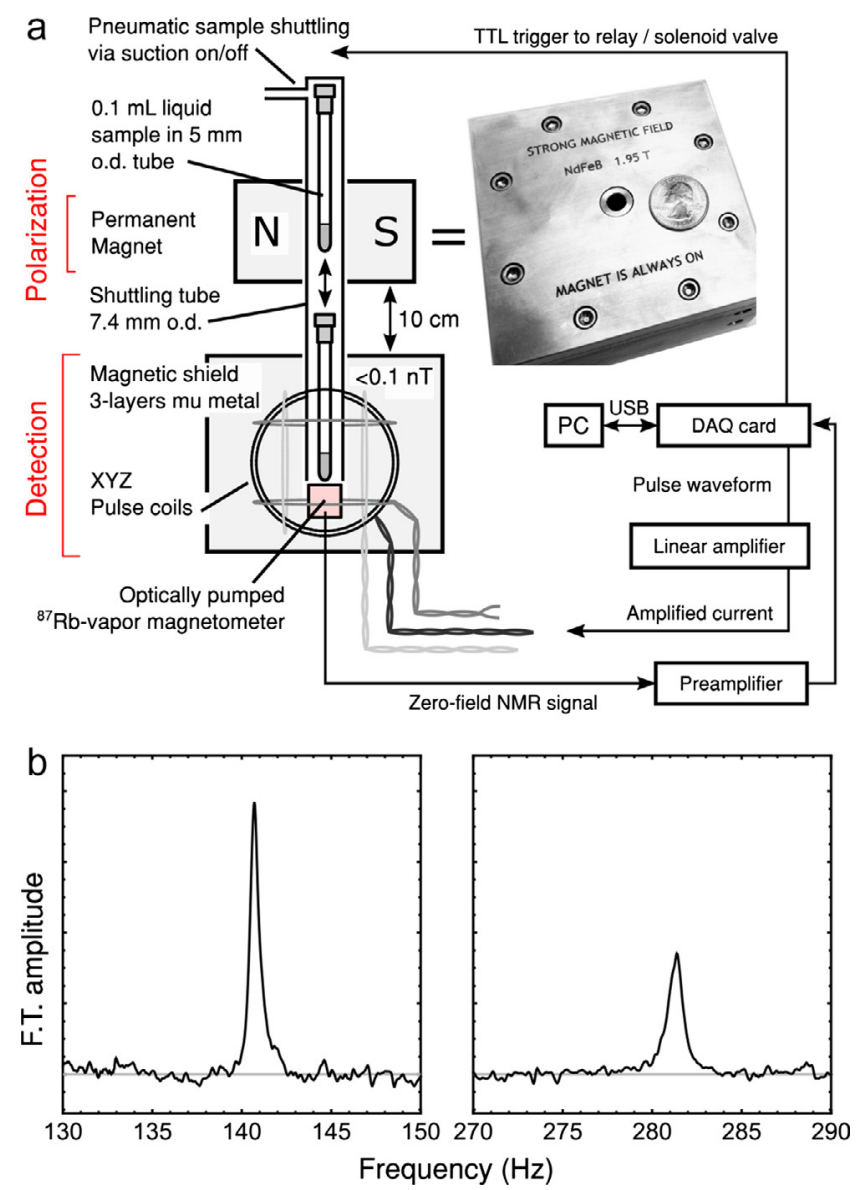

Fig. 6. Experiment used to test the performance of the permanent magnet array for spin polarization in an NMR sample. Part (a) shows a schematic view of a homebuilt zero-field NMR spectrometer based on an optically pumped rubidium magnetometer. The sample, contained in a standard $5 \mathrm{~mm}$ NMR tube, is polarized within the permanent magnet and then shuttled into a magnetic shield where the magnetometer resides. After transport to zero field, a DC pulse excites the spin coherence and the signal is detected by the magnetometer. In (b) we show the spectrum recorded for $0.1 \mathrm{~mL}$ neat $\left[{ }^{13} \mathrm{C}\right]$-methanol, where the signals appear at $1 \times$ and $2 \times$ the scalar ${ }^{1} J_{\mathrm{CH}}$ coupling frequency.

The zero-field NMR spectrum was essentially unchanged, except for a slight increase in signal intensity - around $\sim 6 \%$ - due to the increased strength of the polarization field (6\% between $1.93 \mathrm{~T}$ and $2.05 \mathrm{~T}$ ).

\section{Conclusion}

We have shown how very strong magnets (up to $2 \mathrm{~T}, 7.5 \mathrm{~mm}$ bore) can be easily produced using "off-the-shelf" NdFeB magnetic blocks and simple engineering. This approach can be easily implemented in any laboratory, with particular focus on low-cost portable pre-polarization at a small fraction of the cost of existing commercial products. More complicated designs and modifications to the material composition (e.g. soft-iron poles) could achieve even higher magnetic field strengths over similar sample volumes. Unfortunately, the size and the complexity of the magnet structure increases exponentially with the field strength. The highest magnetic field achieved using permanent magnets presently stands at around $5 \mathrm{~T}$ over volumes of a few microliters $[29,30]$. One should seek a different technology such as high-temperature superconductors for reaching magnetic fields beyond $5 \mathrm{~T}$. Future work may involve measuring the field homogeneity of our magnet, to determine the extent of correction required to measure NMR spectra with resolved chemical shifts.

\section{Acknowledgement}

This work has received support from the European Research Council (author DS, grant agreement FP7-205119 R-EvolutioNMR) and from the European Commission's Seventh Framework Program (author MCDT under the Marie Curie International Outgoing Fellowship Programme, grant agreement FP7-625054 ODMRCHEM; author DS, 2007-2013). We are required to state that the contents of the work do not reflect the views of either the university or the European Commission. We thank Joseph Kant (UCB physics, student machine shop) for discussions and training. Additionally, we greatly thank Alex Pines (UCB) and Dmitry Budker (UCB and Helmholtz Institut Mainz) for support.

\section{Appendix A. Supplementary material}

Supplementary data associated with this article can be found, in the online version, at http://dx.doi.org/10.1016/j.jmr.2017.03.001.

\section{References}

[1] R. Teodorescu, NMR magnets: a historical overview, in: A.J. Williams, G.E. Martin, D. Rovnyak (Eds.), Modern NMR Approaches to the Structure Elucidation of Natural Products, vol. 1, Royal Society of Chemistry, 2016 (Chapter 2).

[2] G. Roth, Ultra-high-field NMR Magnet Design, 2016. <http://www.nmr. bioc.cam.ac.uk/wp-content/uploads/2011/03/bruker magnets.pdf $>$.

[3] P. Campbell, Permanent Magnet Materials and their Applications, Cambridge Univ. Press, 1996.

[4] K. Halbach, Design of permanent multipole magnets with oriented rare earth cobalt material, Nucl. Instrum. Methods 169 (1980) 1-10.

[5] H. Zijlstra, Permanent magnet systems for NMR tomography, Philips J. Res. 40 (1985) 259-288.

[6] G. Moresi, R. Magin, Miniature permanent magnet for table-top NMR, Conc. Magn. Reson. B 19 (2003) 35-43.

[7] B. Manz, M. Benecke, F. Volke, A simple, small and low cost permanent magnet design to produce homogeneous magnetic fields, J. Magn. Reson. 192 (2008) 131-138.

[8] E. Danieli, J. Perlo, B. Blümich, F. Casanova, Small magnets for portable NMR spectrometers, Angew. Chem. Int. Ed. 49 (2010) 4133-4135.

[9] A.E. Marble, I.V. Mastikhin, B.G. Colpitts, B.J. Balcom, A compact permanent magnet array with a remote homogeneous field, J. Magn. Reson. 186 (2007) $100-104$.

[10] C. Hugon, P.M. Aguiar, G. Aubert, D. Sakellariou, Design, fabrication and evaluation of a low-cost homogeneous portable permanent magnet for NMR and MRI, Comp. Rend. Chim. 13 (2010) 388-393.

[11] C. Hugon, F. D’Amico, G. Aubert, D. Sakellariou, Design of arbitrarily homogeneous permanent magnet systems for NMR and MRI: theory and experimental developments of a simple portable magnet, J. Magn. Reson. 205 (2010) 75-85.

[12] D. Sakellariou, C. Hugon, A. Guica, G. Aubert, S. Cazaux, Permanent magnet assembly producing a strong tilted homogeneous magnetic field: towards magic angle field spinning NMR and MRI, Magn. Reson. Chem. 48 (2010) 903908.

[13] P. Poulichet, A. Fakri, C. Delabie, H.D. Phuc, T.T. Cong, L. Fakri-Bouchet, Simulation and optimization of homogeneous permanent magnet for portable NMR applications, in: Proc. Int. Conf. Sens. Tech., 2014.

[14] B.P. Hills, K.M. Wright, D.G. Gillies, A low-field, low-cost Halbach magnet array for open-access NMR, J. Magn. Reson. 175 (2005) 336-339.

[15] G.J. Béné, Nuclear magnetism of liquid systems in the earth field range, Phys. Rep. 58 (4) (1980) 213-267.

[16] M. Burghoff, S. Hartwig, L. Trahms, J. Bernarding, Nuclear magnetic resonance in the nanotesla range, Appl. Phys. Lett. 87 (2005) 054103.

[17] L. Trahms, M. Burghoff, NMR at very low fields, Magn. Reson. Imag. 28 (2010) 1244-1250.

[18] A.F. McDowell, N.L. Adolphi, Operating nanoliter scale NMR microcoils in a 1 tesla field, J. Magn. Reson. 188 (2007) 74-82.

[19] A.F. McDowell, E. Fukushima, Ultracompact NMR: $1 \mathrm{H}$ spectroscopy in a subkilogram magnet, Appl. Magn. Reson. 35 (2008) 185-195.

[20] D. Topgaard, R.W. Martin, D. Sakellariou, C.A. Meriles, A. Pines, “Shim pulses” for NMR spectroscopy and imaging, Proc. Natl. Acad. Sci. USA 101 (2004) $17576-17581$.

[21] H. Raich, P. Blümler, Design and construction of a dipolar Halbach array with a homogeneous field from identical bar magnets: NMR Mandhalas, Conc. Magn. Reson. B 23 (2004) 16-25. 
[22] N. Dogan, R. Topkaya, H. Subaşı, Y. Yerli, B. Rameev, Development of Halbach magnet for portable NMR device, J. Phys. Conf. Ser. 153 (2009) 012047, http:// dx.doi.org/10.1088/1742-6596/153/1/012047.

[23] Q.Y. Chen, G.C. Zhang, Y.J. Xu, Y. Chang, H. Wang, X.D. Yang, Simulation and optimization of an octagonal Halbach permanent magnet array for small-size NMR, Appl. Mech. Mater. 543-547 (2014) 509-513.

[24] C.W. Windt, H. Soltner, D. van Dusschoten, P. Blümler, A portable Halbach magnet that can be opened and closed without force: the NMR-CUFF, J. Magn. Reson. 208 (2011) 27-33.

[25] D. Givord, M. Rossignol, D. Taylor, Coercivity mechanisms in hard magnetic materials, J. Phys. Colloque IV 2 (1992) 95-104, http://dx.doi.org/10.1051/ jp4:1992314.
[26] A.G. Clegg, I.M. Coulson, G. Hilton, H.Y. Wong, The temperature stability of NdFeB and NdFeBCo magnets, IEEE Trans. Magn. 26 (1990) 1942-1944.

[27] J.W. Blanchard, D. Budker, Zero to ultralow-field NMR, eMagRes 5 (2016) 1395-1410.

[28] M.C.D. Tayler, T. Theis, T.F. Sjolander, J.W. Blanchard, A. Kentner, S. Pustelny, A Pines, D. Budker, Instrumentation for nuclear magnetic resonance in zero and ultralow magnetic field, Rev. Sci. Instrum. (2017) (submitted for publication).

[29] F. Bloch, O. Cugat, J.C. Toussaint, G. Meunier, Approches novatrices à génération de champs magnétiques intenses: Optimisation d'une source de flux à aimants permanents, Eur. Phys. J. AP 5 (1999) 85-89.

[30] M. Kumada, E.I. Antokhin, Y. Iwashita, M. Aoki, E. Sugiyama, Super strong permanent dipole magnet, IEEE Trans. Appl. Supercond. 14 (2004) 1287-1289. 